\title{
Clinical Study \\ Fecal Lactoferrin: Reliable Biomarker for Intestinal Inflammation in Pediatric IBD
}

\author{
Stephan Buderus, ${ }^{1}$ James H. Boone, ${ }^{2}$ and Michael J. Lentze ${ }^{3}$ \\ ${ }^{1}$ Department of Pediatrics, St. Marien-Hospital, GFO-Kliniken Bonn, Robert-Koch-Straße 1, 53115 Bonn, Germany \\ ${ }^{2}$ TechLab Inc., Blacksburg, VA 24060, USA \\ ${ }^{3}$ Department of Pediatrics, University Children's Hospital, Adenauerallee 119, 53113 Bonn, Germany
}

Correspondence should be addressed to Stephan Buderus; s.buderus@web.de

Received 26 February 2015; Revised 2 May 2015; Accepted 2 May 2015

Academic Editor: Rami Eliakim

Copyright (C) 2015 Stephan Buderus et al. This is an open access article distributed under the Creative Commons Attribution License, which permits unrestricted use, distribution, and reproduction in any medium, provided the original work is properly cited.

\begin{abstract}
Background. Optimal management of pediatric patients with inflammatory bowel disease (IBD) requires early diagnosis. Aim of the study is to compare fecal lactoferrin (FL) as biomarker of intestinal inflammation to CRP in pediatric patients with new-onset IBD. Methods. FL was measured by ELISA in stool specimens collected prior to endoscopy for IBD (IBD-SCAN; TechLab, Blacksburg; normal $<7.3 \mu \mathrm{g} / \mathrm{g}$ feces). CRP was detected in serum (normal $<5 \mathrm{mg} / \mathrm{L}$ ). Three patient groups were determined: $n=21$ (mean age 13.2) with Crohn's disease (CD), $n=15$ (mean age 10.9) with ulcerative colitis (UC), and $n=20$ (mean age 11.9) in whom IBD was ruled out. In CD patients the endoscopic severity score SES-CD was correlated with the FL levels. Results. (Mean \pm SEM). CRP levels were $27.18 \pm 4.2$ for CD-cases, $20.8 \pm 9.5$ for UC, and $0.24 \pm 0.06$ for non-IBD patients. FL levels were $313.6 \pm 46.4$ in CD, $370.7 \pm 46.9$ in UC, and $1.3 \pm 0.5$ in non-IBD patients. Sensitivity of CRP to detect IBD was $75 \%$ with specificity of $100 \%$, positive predictive value of $100 \%$, and negative predictive value of $69 \%$. Sensitivity of FL was $100 \%$ with specificity of $95 \%$, positive predictive value of $97.3 \%$, and negative predictive value of $100 \%$. In CD, FL levels correlated positively $\left(R^{2}=0.42\right)$ with disease severity as judged by the SES-CD. Conclusions. Elevated FL corresponds to intestinal inflammation, even in patients with normal CRP. With high probability, normal FL excludes intestinal inflammation.
\end{abstract}

\section{Background}

Inflammatory bowel disease (IBD) involves a large clinical spectrum of disease presentations from mild to severe symptoms in relation to different disease locations and the extent from possible rectal to upper intestinal involvement. In pediatric patients, early diagnosis of IBD is of great relevance and essential for best outcome. Induction of remission by specific therapy aims to improve the patient's symptoms, to maintain or restore the quality of life as soon as possible, and to prevent complications of the disease [1-5]. Human lactoferrin, a neutrophil derived glycoprotein, can be measured in feces and whole gut lavage as an indicator of intestinal inflammation in both IBD and infectious gastroenteritis [6,7]. Recent studies have shown fecal lactoferrin (FL) as a sensitive biomarker for pediatric IBD $[8,9]$. In addition, this biomarker can serve as an aid for guiding the diagnostic and therapeutic process for both pediatric and adult IBD [10-12]. In this study, we evaluated the utility of FL compared to CRP $[13,14]$ for diagnosing pediatric IBD characterized by endoscopic and histologic examination. Moreover, we analysed FL in comparison to the disease severity in $\mathrm{CD}$ as defined by the endoscopic score SES-CD [15].

\section{Patients and Methods}

2.1. Patients. In this observational study 56 pediatric patients who qualified for colonoscopy because of symptoms suggestive of IBD were recruited. Ileocolonoscopy with biopsies was performed by an academic teaching hospital, serving as tertiary care pediatric gastroenterology center. As part of the routine clinical assessment, CRP and the FL were determined in all patients prior to endoscopy. Based on results of endoscopy and histology, the diagnosis of IBD was either 
established or ruled out as non-IBD. The IBD patients were further defined as Crohn's disease (CD) or ulcerative colitis (UC). The study was performed with the approval of the local ethical committee.

2.2. Data Collection and Laboratory Analysis. Fecal lactoferrin was determined quantitatively by an ELISA (IBD-SCAN; TechLab, Blacksburg, VA) in stool specimens collected prior to endoscopy as part of the diagnostic workup for IBD. FL results are reported as $\mu \mathrm{g} / \mathrm{g}$ feces (normal $<7.3$ ). SerumCRP was determined by standard methods and is reported as $\mathrm{mg} / \mathrm{L}$ serum (normal $<5$ ). Inflammatory bowel disease was diagnosed and classified according to the "Porto-Criteria," that is, based on the clinical picture, laboratory, and imaging results and most importantly on the results of endoscopy and histology [16]. All patients had an ileocolonoscopy with biopsies taken from each examined segment of the intestine. The macroscopic aspect of the ileocolon was described qualitatively as normal, or showing slight, intermediate, or severe inflammation, respectively; in addition the SES-CD [15] was calculated as endoscopic severity score in CD patients. The score evaluates size of ulcers, ulcerated surface, affected surface, and narrowing in the different segments of the colon and terminal ileum, with a maximal score of 60 . In addition, the distribution of the disease was categorized according to the "Paris Classification," the pediatric modification of the Montreal Classification [17]. According to the PortoCriteria, all patients with new-onset IBD also received an upper endoscopy and small bowel imaging.

2.3. Statistical Analysis. Calculations and plotting of the graphs were performed using GraphPad Prism, version 4.03 for Windows, GraphPad Software, San Diego, California, USA. Results are expressed as mean \pm standard deviation (SD), unless otherwise indicated. In the box and whiskers graphs, the mean is depicted by the line in the box which extends from the 25 th to the 75 th percentile of the data. Significance levels to compare CRP and FL of the IBD patients versus controls were calculated using the two tailed $t$-test, with a $P$ value $<0.05$ set as significant.

\section{Results}

A total of 56 patients with ileocolonoscopies were included in this study (Figure 1). The demographic and clinical characteristics are shown in Table 1 . There were 20 patients with nonIBD illnesses including functional abdominal pain, irritable bowel syndrome, or constipation. For these patients, upper and lower endoscopy and the histology of both examinations were normal with no signs typical for IBD.

3.1. CRP. In the IBD group, the mean CRP levels were elevated with a wide range of concentrations (Figure 2). The mean CRP for CD cases was $27.2 \mathrm{mg} / \mathrm{L}$ (range 0-63.00; SD 19.04); for UC cases, the mean CRP was 20.8 (range 0-145.2; SD 36.63). There were $2 \mathrm{CD}$ (9.5\% of CD) patients with normal CRP. Accordingly, 3 out of 15 UC (20\%) patients had negative CRP. All of the UC patients with CRPs $\geq 16 \mathrm{mg} / \mathrm{L}$

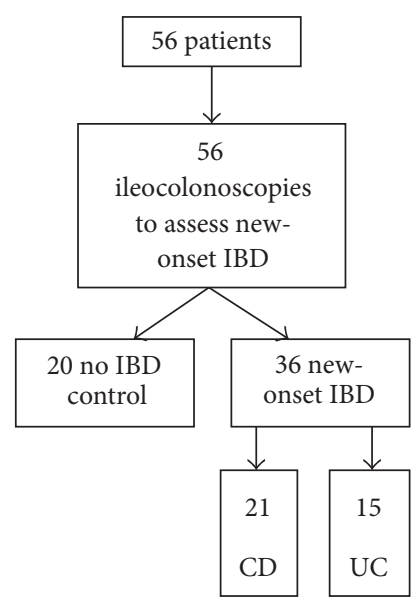

FIGURE 1: Patient and endoscopy flowchart.

TABLE 1: Patient and clinical characteristics.

\begin{tabular}{lccc}
\hline Group & CD & UC & Control \\
\hline$n$ & 21 & 15 & 20 \\
Male/female & $15 / 6$ & $10 / 5$ & $10 / 10$ \\
Mean/median age & $13.2 / 13.4$ & $10.9 / 12.7$ & $11.9 / 12.6$ \\
Paris classification & & & \\
$\quad$ CD L1 & 4 & & \\
CD L2 & 4 & & \\
CD L3 & 13 & & \\
UC E3 & & 1 & \\
UC E4 & & 14 & \\
\hline
\end{tabular}

suffered from severe pancolitis. None of the non-IBD patients had elevated CRP with a mean of $0.24 \mathrm{mg} / \mathrm{L}$ (SD 0.28) and a maximum of 0.8 (Figure 2). Sensitivity of CRP to detect IBD was $75 \%$ with a specificity of $100 \%$, correspondingly resulting in a positive predictive value of $100 \%$ and negative predictive value of $69 \%$; the negative likelihood ratio (LR) of CRP is 0.25 .

3.2. Fecal Lactoferrin (FL). The mean levels of FL for the IBD patients were $314 \mu \mathrm{g} / \mathrm{g}$ CD (SD 212.8) and $371 \mu \mathrm{g} / \mathrm{g}$ UC (SD 181.5) whilst mean FL in controls was only 1.3 (SD 2.4) (Figure 3). Only a single non-IBD-patient had an elevated FL of $9.6 \mu \mathrm{g} / \mathrm{g}$ feces, slightly above the clinical cut-off of $7.3 \mathrm{ug} / \mathrm{g}$. Therefore, sensitivity of FL was $100 \%$ with a specificity of $95 \%$, the positive predictive value being $97.3 \%$ and having a negative predictive value of $100 \%$. The positive LR for FL is 20.0 , whilst the negative LR is 0 . For the CD patients the results of the endoscopic severity index SES-CD in correlation with the corresponding FL levels are depicted in Figure 4. There is a positive correlation of CD-SES and FL, showing higher levels of FL $\left(R^{2}=0.42\right.$ and $\left.P=0.014\right)$ in those patients with a more severe disease as judged by endoscopy.

\section{Discussion}

These results demonstrate the value of determining fecal lactoferrin levels as an aid for diagnosing IBD in pediatric 


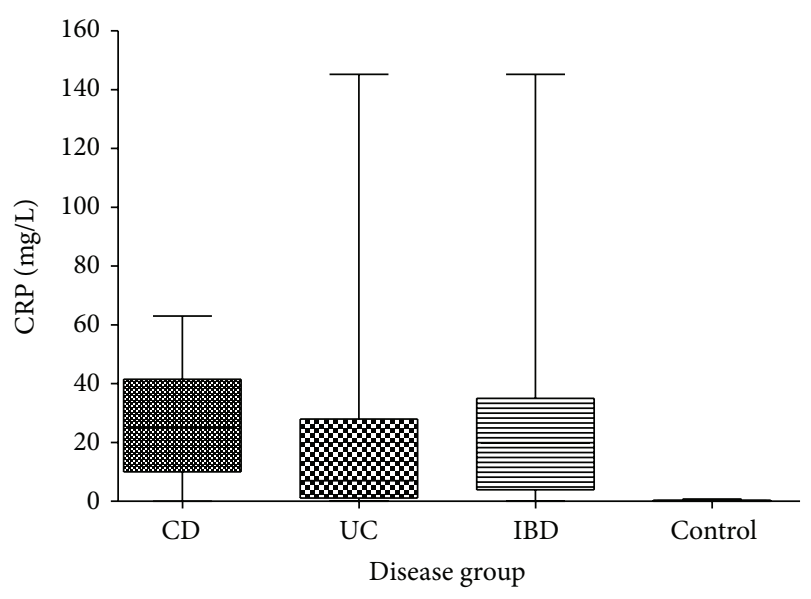

FIgURE 2: Serum-CRP levels stratified for groups of patients. $P<$ 0.0001 IBD versus control and CD versus control. $P=0.017$ UC versus control. $P=0.50$ for $\mathrm{CD}$ versus $\mathrm{UC}$.

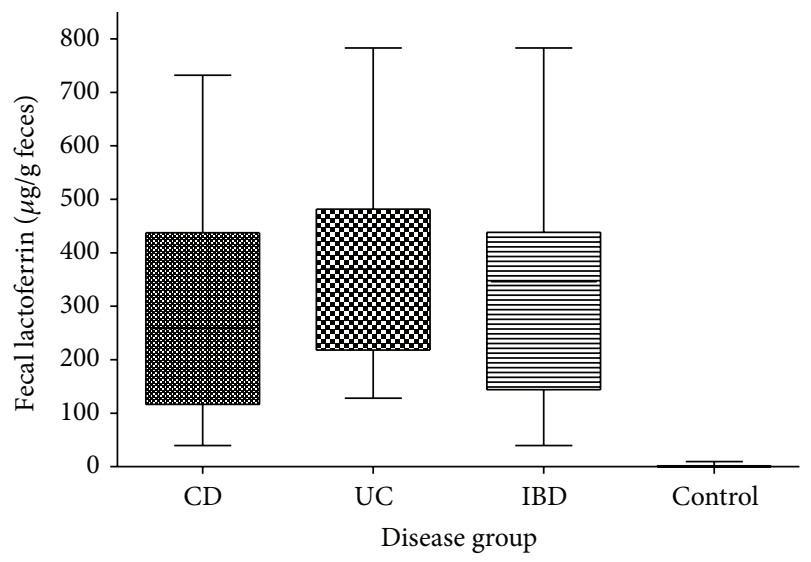

FIGURE 3: Fecal lactoferrin levels stratified for groups of patients. $P<$ 0.0001 for each IBD, CD, and UC versus control. $P=0.41$ for CD versus UC.

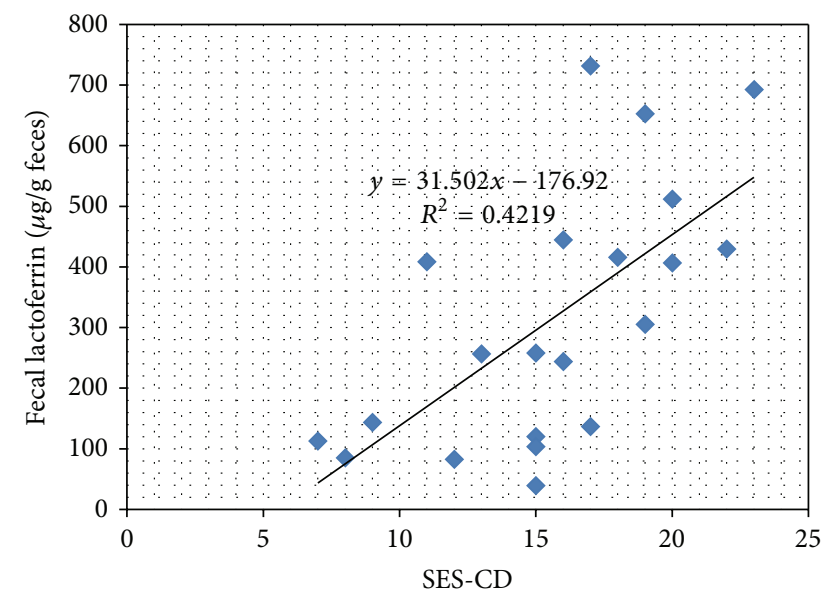

$\checkmark$ FLA

— Linear (FLA)

FIGURE 4: CD patients: fecal lactoferrin and the endoscopic score $\mathrm{SES}-\mathrm{CD}$, linear regression line depicted in the graph. patients and correlate levels with disease severity as judged by the SES-CD in CD patients. Elevated FL ( $\geq 7.3 \mathrm{ug} / \mathrm{g}$ feces) differentiated precisely between subjects with the presence of intestinal inflammation and those having intact and normal intestinal; this is underscored by the high positive (20) and low (0) negative LR of FL. Patients with normal FL had no evidence of microscopic or histologic intestinal inflammation. This is in accordance with other pediatric and adult studies $[7-9,11]$. The presence of elevated LF in patients with normal CRP levels during active disease shows the inferiority of this parameter in comparison to FL to detect IBD. Currently, serum-CRP is an inflammatory serological marker that is routinely measured in a number of clinical situations, among them IBD. The strength of CRP is that every physician can easily and rapidly obtain a result as a routine test. Previous studies have shown $[13,14,18]$ that, in CD, CRP is useful in identifying new patients with active disease and monitoring a response to therapy. A limitation for CRP measurements is that levels may be normal in up to $24-64 \%$ of pediatric IBD patients experiencing a flare, making it insufficient for excluding active IBD. This corresponds well to our findings that $10 \%$ of our active CD patients and $20 \%$ of the UC patients were CRP-negative.

A new finding from this study is the observation that the levels of LF correlate with disease severity. In CD patients grouped by SES-CD those with more severe disease had significantly higher LF levels than patients with less inflammation. In UC, we could not observe significant differences between LF levels as all patients had extensive colitis (Paris E4 and Paris E3).

A weakness of the study with respect to parameters such as sensitivity or specificity is the fact the study group is preselected for suspicion of IBD in a tertiary center. This means that those parameters might be lower in a less restricted patient group. But we have clearly shown in a relatively large control group with symptoms severe enough to justify endoscopy that normal FL excludes intestinal inflammation caused by IBD with high probability $(P<0.0001)$. Clinically, in the pediatric setting of many patients with functional abdominal pain or irritable bowel syndrome [19-21] and in comparison to those relatively few IBD patients, elevated FL can help to define those patients who should undergo endoscopy and those who do not need invasive diagnostics.

\section{Conclusion}

Fecal lactoferrin is a reliable biomarker for active IBD in pediatric patients. At least for patients with CD, FL levels are correlated with disease severity as graded by the SES-CD. The serological marker CRP is less sensitive for active IBD. Thus, FL is a helpful biomarker to aid the early diagnosis of pediatric IBD, indicating the need for invasive diagnostics like endoscopy. It shows promise for monitoring the effectiveness of therapy because of its correlation of levels and degree of mucosal inflammation. 


\section{Conflict of Interests}

S. Buderus received study material (assays) from TechLab Inc. and travel grants to present results on scientific meetings. J. Boone is Senior Research Scientist, Research and Development at TechLab, Inc. M. J. Lentze has no disclosures.

\section{Authors' Contribution}

Stephan Buderus designed the study and took care of the patients and the samples. He drafted the paper. James $\mathrm{H}$. Boone was involved in the design of the study; he was involved in the statistical analysis and the drafting of the paper. Michael J. Lentze was involved in the design of the study and the drafting of the paper. All authors read and approved the final paper.

\section{Acknowledgments}

The authors thank H. Ringelmann and T. Rottman for their dedicated performance in analyzing fecal lactoferrin.

\section{References}

[1] S. Auvin, F. Molinié, C. Gower-Rousseau et al., "Incidence, clinical presentation and location at diagnosis of pediatric inflammatory bowel disease: a prospective population-based study in northern France (1988-1999)," Journal of Pediatric Gastroenterology \& Nutrition, vol. 41, no. 1, pp. 49-55, 2005.

[2] E. I. Benchimol, A. Guttmann, A. M. Griffiths et al., "Increasing incidence of paediatric inflammatory bowel disease in Ontario, Canada: Evidence from health administrative data," Gut, vol. 58, no. 11, pp. 1490-1497, 2009.

[3] H. Hildebrand, Y. Finkel, L. Grahnquist, J. Lindholm, A. Ekbom, and J. Askling, "Changing pattern of paediatric inflammatory bowel disease in northern Stockholm 1990-2001," Gut, vol. 52, no. 10, pp. 1432-1434, 2003.

[4] S. Kugathasan, R. H. Judd, R. G. Hoffmann et al., "Epidemiologic and clinical characteristics of children with newly diagnosed inflammatory bowel disease in Wisconsin: a statewide population-based study," Journal of Pediatrics, vol. 143, no. 4, pp. 525-531, 2003.

[5] K. Eszter Müller, P. Laszlo Lakatos, M. Papp, and G. Veres, "Incidence and paris classification of pediatric inflammatory bowel disease," Gastroenterology Research and Practice, vol. 2014, Article ID 904307, 10 pages, 2014.

[6] S. V. Kane, W. J. Sandborn, P. A. Rufo et al., "Fecal lactoferrin is a sensitive and specific marker in identifying intestinal inflammation," American Journal of Gastroenterology, vol. 98, no. 6, pp. 1309-1314, 2003.

[7] J. Langhorst, S. Elsenbruch, J. Koelzer, A. Rueffer, A. Michalsen, and G. J. Dobos, "Noninvasive markers in the assessment of intestinal inflammation in inflammatory bowel diseases: performance of fecal lactoferrin, calprotectin, and PMN-elastase, CRP, and clinical indices," American Journal of Gastroenterology, vol. 103, no. 1, pp. 162-169, 2008.

[8] T. R. Walker, M. L. Land, A. Kartashov et al., "Fecal lactoferrin is a sensitive and specific marker of disease activity in children and young adults with inflammatory bowel disease," Journal of Pediatric Gastroenterology and Nutrition, vol. 44, no. 4, pp. 414422, 2007.
[9] M. D. Pfefferkorn, J. H. Boone, J. T. Nguyen, B. E. Juliar, M. A. Davis, and K. K. Parker, "Utility of fecal lactoferrin in identifying crohn disease activity in children," Journal of Pediatric Gastroenterology and Nutrition, vol. 51, no. 4, pp. 425-428, 2010.

[10] S. Buderus, J. Boone, D. Lyerly, and M. J. Lentze, "Fecal lactoferrin: a new parameter to monitor infliximab therapy," Digestive Diseases and Sciences, vol. 49, no. 6, pp. 1036-1039, 2004.

[11] T. Sipponen, E. Savilahti, K.-L. Kolho, H. Nuutinen, U. Turunen, and M. Färkkilä, "Crohn's disease activity assessed by fecal calprotectin and lactoferrin: correlation with Crohn's disease activity index and endoscopic findings," Inflammatory Bowel Diseases, vol. 14, no. 1, pp. 40-46, 2008.

[12] J. P. Gisbert, A. G. McNicholl, and F. Gomollon, "Questions and answers on the role of fecal lactoferrin as a biological marker in inflammatory bowel disease," Inflammatory Bowel Diseases, vol. 15, no. 11, pp. 1746-1754, 2009.

[13] M. Sidoroff, R. Karikoski, T. Raivio, E. Savilahti, and K.L. Kolho, "High-sensitivity C-reactive protein in paediatric inflammatory bowel disease," World Journal of Gastroenterology, vol. 16, no. 23, pp. 2901-2906, 2010.

[14] A. Tsampalieros, A. M. Griffiths, N. Barrowman, and D. R. MacK, "Use of C-reactive protein in children with newly diagnosed inflammatory bowel disease," The Journal of Pediatrics, vol. 159, no. 2, pp. 340-342, 2011.

[15] M. Daperno, G. D’Haens, G. van Assche et al., "Development and validation of a new, simplified endoscopic activity score for Crohn's disease: the SES-CD," Gastrointestinal Endoscopy, vol. 60, no. 4, pp. 505-512, 2004.

[16] A. Levine, S. Koletzko, D. Turner et al., "ESPGHAN revised porto criteria for the diagnosis of inflammatory bowel disease in children and adolescents," Journal of Pediatric Gastroenterology and Nutrition, vol. 58, no. 6, pp. 795-806, 2014.

[17] A. Levine, A. Griffiths, J. Markowitz et al., "Pediatric modification of the Montreal classification for inflammatory bowel disease: the Paris classification," Inflammatory Bowel Diseases, vol. 17, no. 6, pp. 1314-1321, 2011.

[18] J. C. Cabrera-Abreu, P. Davies, Z. Matek, and M. S. Murphy, "Performance of blood tests in diagnosis of inflammatory bowel disease in a specialist clinic," Archives of Disease in Childhood, vol. 89, no. 1, pp. 69-71, 2004.

[19] M. Saps, M. Sztainberg, and C. di Lorenzo, "A prospective community-based study of gastroenterological symptoms in school-age children," Journal of Pediatric Gastroenterology and Nutrition, vol. 43, no. 4, pp. 477-482, 2006.

[20] P. G. Ramchandani, M. Hotopf, B. Sandhu, and A. Stein, “The epidemiology of recurrent abdominal pain from 2 to 6 years of age: results of a large, population-based study," Pediatrics, vol. 116, no. 1, pp. 46-50, 2005.

[21] D. K. Chitkara, N. J. Talley, A. L. Weaver et al., "Incidence of presentation of common functional gastrointestinal disorders in children from birth to 5 years: a cohort study," Clinical Gastroenterology and Hepatology, vol. 5, no. 2, pp. 186-191, 2007. 


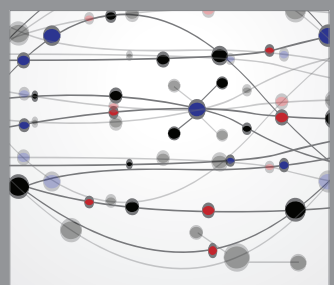

The Scientific World Journal
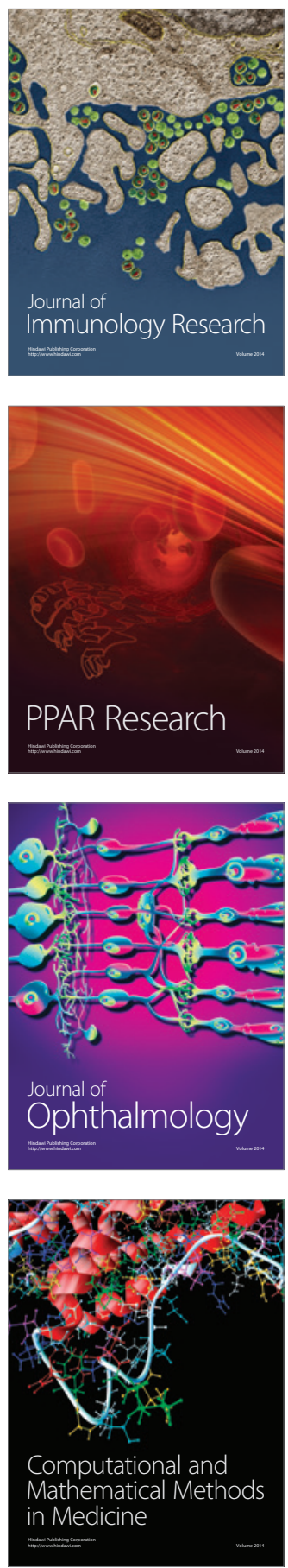

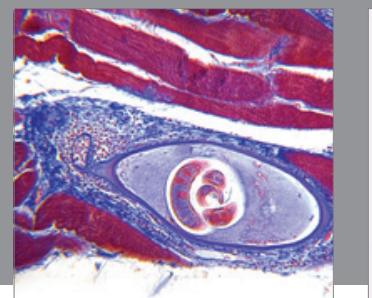

Gastroenterology

Research and Practice
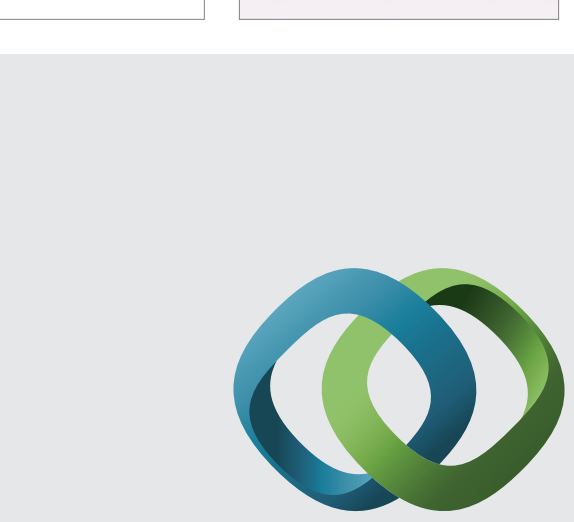

\section{Hindawi}

Submit your manuscripts at

http://www.hindawi.com
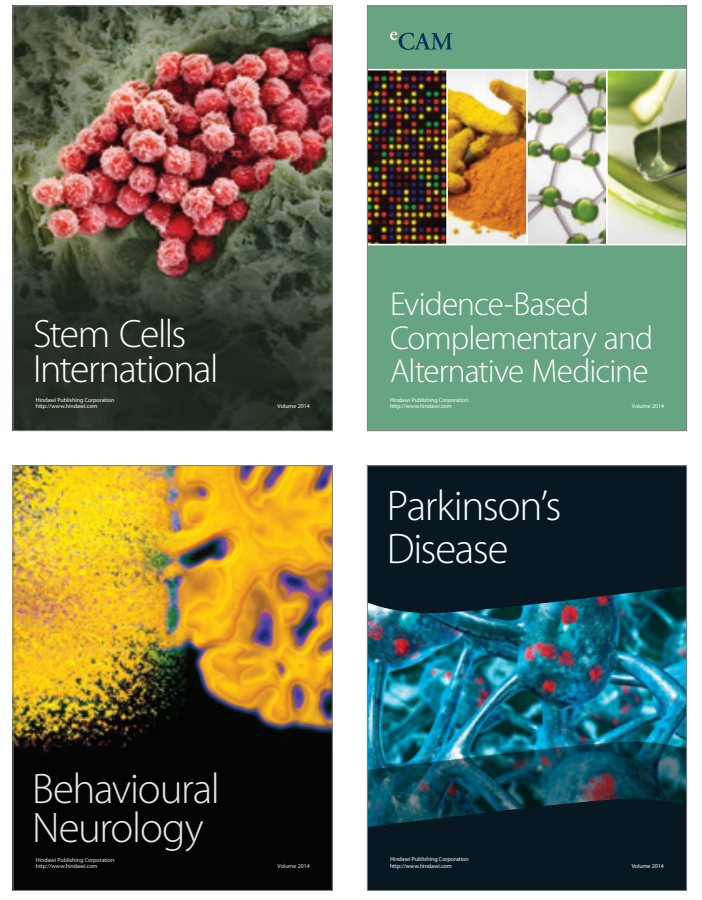
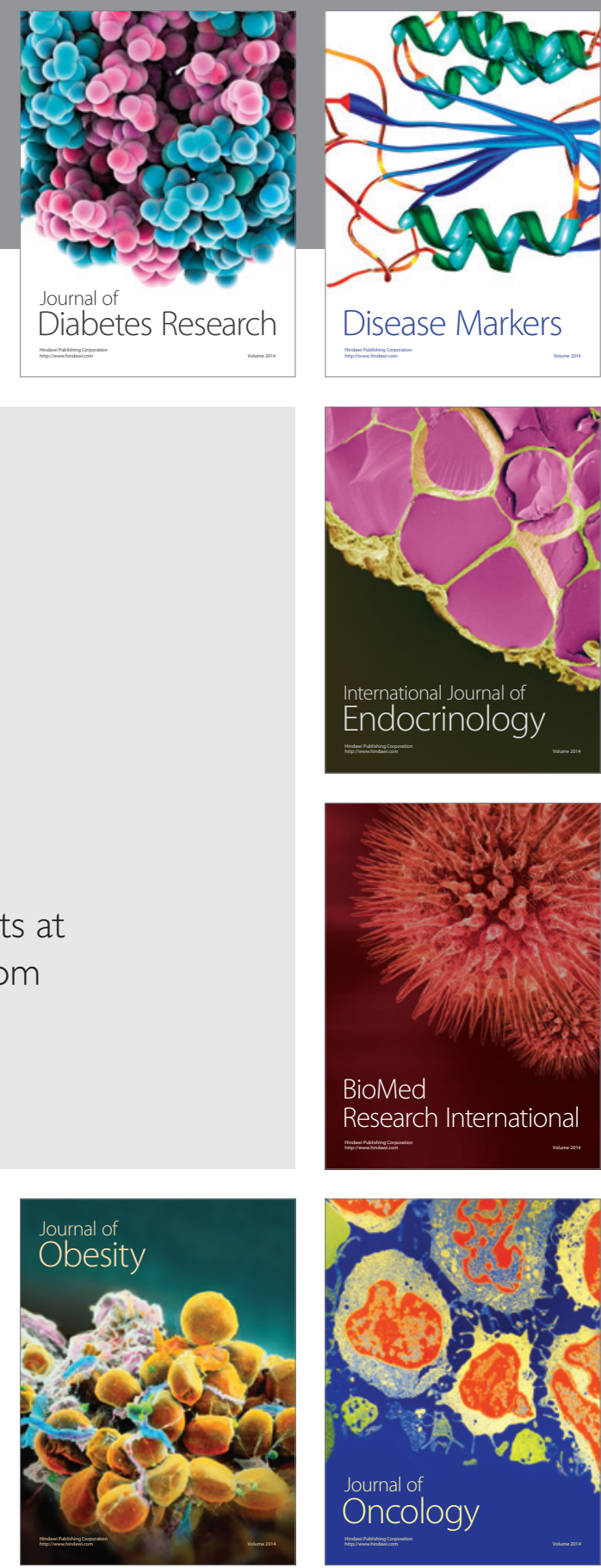

Disease Markers
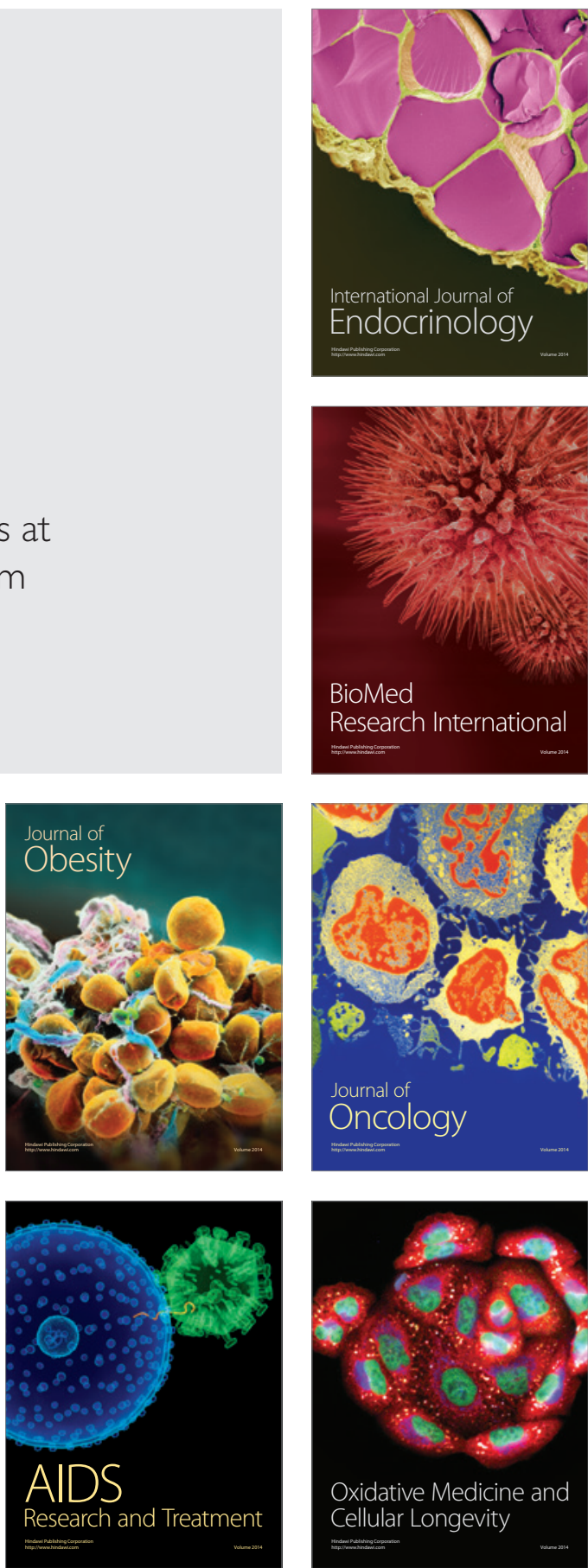